Ophthalmologe 2010 $\cdot 107: 605$

DOI 10.1007/s00347-009-2105-z

Online publiziert: 16. April 2010

๑) Springer-Verlag 2010

F. Stehr

NCL-Stiftung, Hamburg

\title{
Neuronale Zeroidlipofuszinose - nicht nur eine Erkrankung der Netzhaut
}

burg und Göttingen existieren entsprechende NCL-Zentren.

Für Augenärzte ist v. a. die juvenile Form (CLN3, JNCL) von Bedeutung, da die Verschlechterung der Sehkraft als Erstes bemerkt wird. Die Visusminderung und anschließende Einengung des Gesichtsfelds führen innerhalb weniger Jahre zur Erblindung. Die Funduskopie zeigt initial häufig eine Schießscheibenmakulopathie. Aber NCL ist nicht nur eine Netzhauterkrankung, denn aufgrund des sukzessiven Absterbens von Nervenzellen findet parallel ein demenzieller Abbau statt. Später kommt es zu epileptischen Anfällen und spastischen Lähmungen, die zur Bettlägerigkeit führen. Die Patienten werden nur in Ausnahmefällen älter als 30 Jahre.

\section{จ Für Augenärzte ist die juvenile Form von Bedeutung}

Eine frühe Diagnose ist sehr wichtig, obwohl noch keine Therapie vorhanden ist, da Familien von einer genetischen Prädisposition wissen sollten. Jüngere Geschwister können vor Auftreten von Symptomen untersucht werden. Auch eine pränatale Diagnostik ist durchführbar. Außerdem werden Fehlbehandlungen bei den $\mathrm{Pa}$ tienten vermieden, und die Ausnutzung palliativer Behandlungsmöglichkeiten ist möglich.

Aufgrund des Fehlens eines profitablen Absatzmarkts forscht die Pharmaindustrie nicht an NCL, daher hat ein betroffener Vater 2002 die gemeinnützige NCL-Stiftung gegründet. Schwerpunkte liegen in - der Aufklärungsarbeit bei relevanten

\footnotetext{
Ärztegruppen,
}

- der Vernetzung von Forschern,

- der kooperativen Förderung von jungen Wissenschaftlern,

- der Forschungsinitiierung, um Therapiestrategien zu entwickeln.

Die Stiftung fungiert als neutrale Anlaufstelle für Mediziner und Wissenschaftler. Es werden verschiedene Fortbildungsmaßnahmen entwickelt und umgesetzt. Bisher haben aufgrund der Stiftungsaktivitäten $25 \%$ der deutschen und ca. $40 \%$ der österreichischen Augenkliniken NCL bei internen Fortbildungsmaßnahmen berücksichtigt. Um der Differenzialdiagnostik mehr Gewicht zu verleihen, wird die NCL-Stiftung zusammen mit Pro Retina ein Plakatprojekt zu diesem Thema starten.

Unser besonderer Dank gilt dem Herausgeber für die Vergabe eines solchen Leitthemas sowie den Autoren. Wir hoffen, hiermit zur Früherkennung von NCL beizutragen.

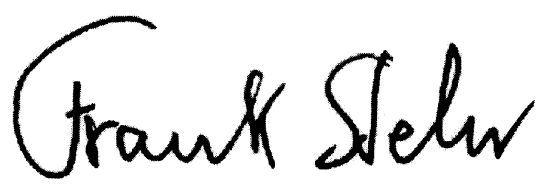

F. Stehr

\section{Korrespondenzadresse}

Dr. F. Stehr

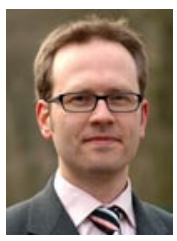

NCL-Stiftung

Holstenwall 10, 20355 Hamburg

frank.stehr@ncl-stiftung.de kommt dem Neuropädiater eine wichtige Rolle zu, da er häufig die Patienten medizinisch bis zu ihrem Tod begleitet. An den Universitätskliniken in Ham- 